\title{
Claudins and Intestinal Barrier Function; The Positive Side
}

\author{
Sabah Nisar", Sheema Hashem" ${ }^{\#}$ and Ajaz A Bhat* \\ Translational Medicine, Qatar \\ *Corresponding author: Ajaz A Bhat, Translational Medicine, Research Branch, Sidra Medicine, Qatar \\ "Both authors contributed equally
}

\begin{tabular}{|c|c|}
\hline ARTICLE INFO & ABSTRACT \\
\hline Received: 慧 September 22, 2019 & Epithelial cell polarity is defined, in part, by Apical Junctional Complex (AJC) that \\
\hline Published: 幽 September 30, 2019 & $\begin{array}{l}\text { separates the plasma membrane into the apical domain and the basolateral domain. } \\
\text { Tight junctions are integral to AJC and form the border between the apical and }\end{array}$ \\
\hline $\begin{array}{l}\text { Citation: Sabah Nisar, Sheema Hashem, } \\
\text { Ajaz A Bhat. Claudins and Intestinal Barrier } \\
\text { Function; The Positive Side. Biomed J Sci \& } \\
\text { Tech Res 21(4)-2019. BJSTR. MS.ID.003637. }\end{array}$ & $\begin{array}{l}\text { cell polarity. Tight junctions also play an important role in cell signaling mechanisms } \\
\text { essential for cell proliferation, migration, differentiation and survival. The claudins, } \\
\text { which constitute a family of at least } 24 \text { members, are the main structural components } \\
\text { of the tight junctions, expressed in a tissue-specific manner and their composition } \\
\text { determines the ion selectivity of the paracellular pathway. Any alteration or disruption } \\
\text { in claudins leads to disorder of epithelial cell polarity and defects in junction formation } \\
\text { or maintenance which in turn results in various pathophysiological conditions like } \\
\text { inflammation and cancer. This mini review discusses the positive relationship between } \\
\text { claudins and intestinal barrier function under normal physiological conditions and how } \\
\text { the dysregulation/mislocalization of claudins can affect the intestinal permeability } \\
\text { under pathological conditions. }\end{array}$ \\
\hline
\end{tabular}

\section{Introduction}

\section{Background}

The intestinal barrier is a thick lining of the gut that is composed of different types of epithelial cells. These epithelial cells act as selective barriers between internal and external cellular environments that allows control of pathogens and other foreign substances. Epithelial cells also control selective movement of ions and solutes. Underneath the epithelial layer, there is a thin layer of connective tissue known as the lamina propria which separates the innermost epithelial cells from the smooth muscle tissue called muscularis mucosa [1]. The epithelial barrier is composed of Tight Junctions (TJ) and adherens junctions, collectively known as Apical Junctional Complex (AJC). AJC is a multifunctional apparatus that plays an important role in cell polarization and tissue differentiation [2] and regulates the structure and function of the cell [3]. Most importantly, the AJC provides strong adhesion between adjacent cells.

Other intercellular junctions that reside below the AJC are desmosomes and gap junctions. These intercellular junctions mediate intercellular adhesion and allow cross talk between adjacent epithelial cells $[4,5]$. The immune cells such as exudate macrophages, eosinophils, neutrophils, eosinophils, and T- cells, help in eliminating antigens and potential pathogens that are resistant to the effector mechanisms of the resident cells of the local immune system and aid in the orchestration of local immune response in the intestinal mucosa [6]. A dense layer of mucus layer that covers the intestinal epithelium prevents the translocation of pathogenic microorganisms and gut microbiota in the underlying intestinal tissues $[7,8]$. and the absence of mucin, a glycoprotein in the mucus layer can lead to intestinal inflammation that predispose an Individual to Various Gastrointestinal (GI) diseases and promote the development of colorectal cancer [9].

Claudins along with occludins are large family of transmembrane proteins that are important components of the TJs. As TJs are the vital components of the epithelial barrier, they are under a constant threat from pro-inflammatory mediators and pathogenic bacteria and viruses [10]. Different claudins perform different 
roles in maintaining the integrity of the intestinal epithelium. Any alteration in the levels of claudins can result in the disruption of the intestinal barrier integrity. Claudins are made up of two extracellular loop domains with $\mathrm{N}$ - and C-terminals and they have the ability to oligomerize in cis- and trans- configuration in order to from diverse combinations $[11,12]$. Claudins are grouped into two categories known as tight claudins (claudin 1, 3, 4, 5 and 18) and leaky claudins (claudin 2, 10 and 15) based on their epithelial permeability [13].

Tight claudins control the adhesion and tightness of the intestinal barrier, while leaky claudins control paracellular permeability [13]. Claudins 3,4 and 7 are expressed at the luminal surface while claudins 2, 10, 13 and 15 are expressed in the crypt-luminal axis $[14,15]$. As these intestinal claudins are involved in the integrity of the intestinal barrier, disruption of the claudins can result in mucosal inflammation leading to inflamed gut. The disruption of the intercellular adhesions between cells can also result in an increase in the epithelial cell motility and migration of mesenchymal cells, a process known as epithelial Mesenchymal Transition (EMT) [16] (Figure 1). This transition causes a decrease in epithelial markers such as E-cadherin and increase in mesenchymal markers such as N-cadherin, vimentin, snail, slug, ZEB-1 and Twist [17]. Claudins play a vital role in the EMT process. Claudin-4 is considered as the main marker of EMT as it increases the number of TJ strands and trans-epithelial resistance but decreases $\mathrm{Na}+$ ion permeability [17]. The alteration of claudin-7 has been shown to disturb the epithelial homeostasis and cause neonatal death in mice and loss of claudin-7 induces EMT and promotes colon cancer [18].

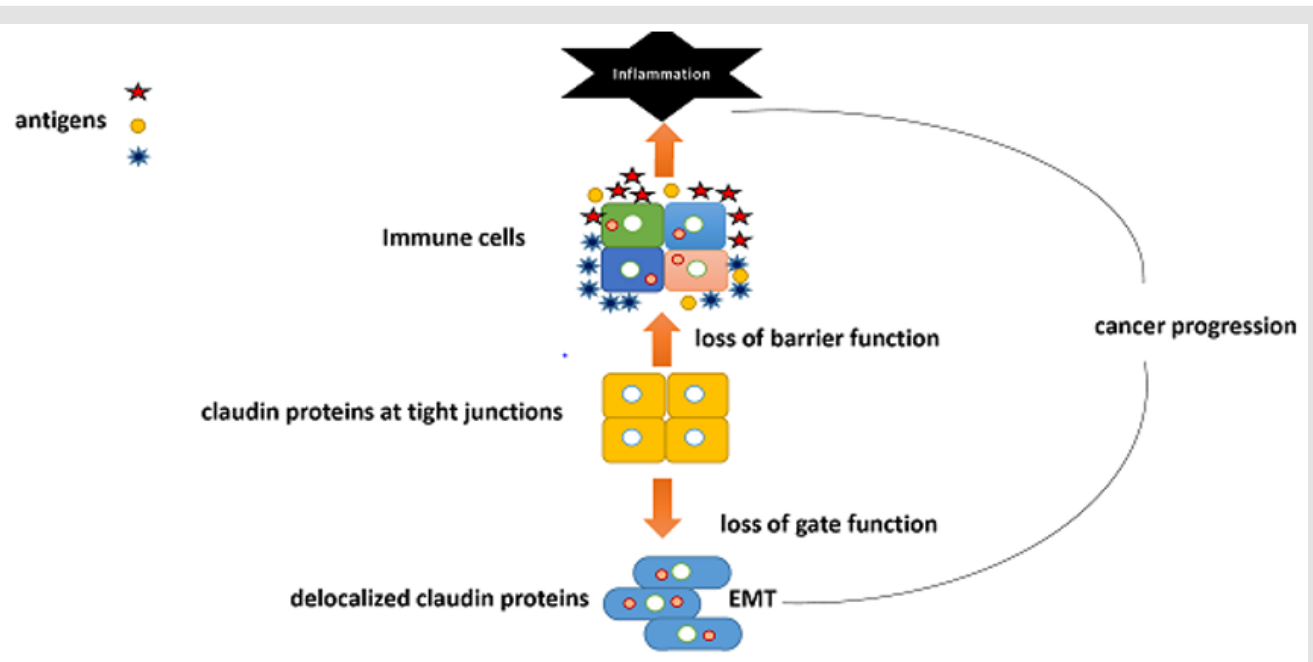

\section{Figure 1}

Figure 1: Claudins are expressed at the site of tight junctions localized on the apical cell membrane. Claudins regulate the gate function and barrier function of tight junctions under normal physiological conditions. However, during cancer development/ progression, tight junctions are often lost, or claudins translocate to other cellular compartments. This facilitates malignant transformation and EMT.

Claudins not only reside in TJs but some claudins such as claudin $1,3,4,5$ and 7 also localize in the plasma membrane of enterocytes [19]. There is little information about the function of non-junctional claudins, but studies have shown that they might play a role in the extracellular matrix and could be involved in maintaining epithelial homeostasis $[19,20]$. Role of different claudin isoforms have been discussed in various studies. As claudins are important in maintaining the intestinal homeostasis, claudin-1 has been found to regulate intestinal epithelial homeostasis through modulation of Notch signaling [21]. Claudin-1 also plays an important role in the regulation of cellular transformation and metastatic behavior in colon cancer and altered expression of claudin-1 has been found to have significant effects on the growth of xenograft tumors in athymic mice [22]. A study found that the integrity of intestinal barrier is reduced by the downregulation of claudin 5 and 8 [23].

While the expression of claudin-2, which is involved in the formation of paracellular water channels is upregulated in inflammatory bowel diseases (IBDs) and promotes inflammation process [24]. Studies have also shown that claudin-7 deficient mice exhibited intestinal defects such as mucosal alterations and inflammation with high production of cytokines [25]. and also increased the paracellular flux of small organic solutes in the intestinal epithelial cells of mice [26]. On the other hand, increased expression of claudin-2 in villin-claudin-2 transgenic mice resulted in increased mucosal permeability and colon length but despite having a leaky colon, claudin- 2 protected the mice from experimentally induced colitis [24]. Claudin-2 is also found to decrease paracellular $\mathrm{Na}+$ permeability in the small intestine of claudin-2 knockout mice [27]. Another study demonstrated that claudin-15 knockout mice developed intestinal abnormalities causing increased disassembly of TJs and enhanced proliferation of cryptic cells resulting in the formation of mega intestine [28].

Within the GI tract, some of the pathogenic bacteria such as Clostridium perfringens enterotoxin (CPE) invade the epithelial 
cells of ileum by binding with specific claudins such as claudin 3and 4 resulting in the disassembly of TJs $[29,30]$. The C-Terminal Region of Cpe (cCPE) binds with claudin-3 and 4 with high affinity and although the CCPE region is not cytotoxic, it acts as a modulator of TJs and its recombinant cCPE proteins can be used for drug delivery and in treatment of tumors that overexpress claudins [31]. The cCPE is also found to form hydrophobic and hydrophilic interactions with the extracellular segments of claudin-19 [32]. Claudins are also involved in certain virus infections such as claudin-1 is an essential component that is required for hepatitis $\mathrm{C}$ virus (HCV) infection as it allows the entry of HCV and is the first factor to be compromised during HCV infection [33]. On the other hand, Helicobacter pylori induces the loss of claudins 4 and 5 from gastric epithelial TJs resulting in increased epithelial permeability [34]. Claudins are also responsible for the phosphorylation of Ephrin B1 by cell-cell contact. Ephrin B1 forms an in vivo complex with adjacent claudin-1 or claudin-4 through binding with their extracellular domains and overexpression of Ephrin B1 altered the cell-cell adhesion in epithelial and cancer cells [35].

Apart from the many functions of claudins, they can be also used as non-invasive biomarkers for intestinal barrier function [36]. They are used as urinary biomarkers such as claudin-3 levels are higher in urine levels in conditions like hemorrhagic shock, IBD and necrotizing enterocolitis [37]. Claudins can be also used as targets for the visualization of different tumors such as claudin-1 is used as target for near infrared (NIR)-antibody based imaging for the purpose of visualizing primary and metastatic colorectal tumors [38]. Claudins are an integral part of the TJs that modulate barrier function, inflammation and regulate solute transport in the GI tract to maintain intestinal homeostasis. Disruption or abnormal expression of claudins can result in various pathophysiological conditions. Claudins can also be promising drug targets that can be used for the development of claudin-based drugs. Claudins can also serve as promoters in mucosal healing by restoring TJs but awaits further research. By exploring various methods that help in the regulation of claudins by different molecules or chemical agents can help in enhancing the intestinal permeability and can aid in the treatment of intestinal diseases and various cancers.

\section{References}

1. Rao J, Wang J (2010) Intestinal Architecture and Development, in Regulation of Gastrointestinal Mucosal Growth. Morgan \& Claypool Life Sciences: San Rafael (CA).

2. Tepass U (2003) Claudin complexities at the apical junctional complex. Nature Cell Biology 5: 595-597.

3. Vogelmann R, WJ Nelson WJ (2005) Fractionation of the epithelial apical junctional complex: reassessment of protein distributions in different substructures. Molecular biology of the cell 16(2): 701-716.

4. Garcia Hernandez V, Quiros M, Nusrat A (2017) Intestinal epithelial claudins: expression and regulation in homeostasis and inflammation. Annals of the New York Academy of Sciences 1397(1): 66-79.

5. Tsukita S, Furuse M, Itoh M (2001) Multifunctional strands in tight junctions. Nature Reviews Molecular Cell Biology 2(4): 285-293.
6. Schenk M, Mueller C (2008) The mucosal immune system at the gastrointestinal barrier. Best Practice \& Research Clinical Gastroenterology 22(3): 391-409.

7. Schroeder BO (2019) Fight them or feed them: how the intestinal mucus layer manages the gut microbiota. Gastroenterology report 7(1): 3-12.

8. Yu Y, Sitaraman, Gewirtz AT (2004) Intestinal epithelial cell regulation of mucosal inflammation. Immunologic Research 29(1): 55-67.

9. Chelakkot C, Ghim J, Ryu SH (2018) Mechanisms regulating intestinal barrier integrity and its pathological implications. Experimental \& Molecular Medicine 50(8): 103.

10. Bhat AA, Shanmugakonar M, Al Naemi HA, Haris M, Uddin S, et al. (2019) Tight Junction Proteins and Signaling Pathways in Cancer and Inflammation: A Functional Crosstalk. Frontiers in physiology 9: 19421942.

11. Overgaard CE, Daugherty BL, Mitchell LA, Koval M (2011) Claudins: control of barrier function and regulation in response to oxidant stress. Antioxidants \& redox signaling 15(5): 1179-1193.

12. Koval M (2013) Differential pathways of claudin oligomerization and integration into tight junctions. Tissue barriers 1(3): e24518-e24518.

13. Anderson JM, Van Itallie CM (2009) Physiology and Function of the Tight Junction. Cold Spring Harbor Perspectives in Biology 1(2).

14. Ivanov AI, Nusrat, Parkos CA (2004) Endocytosis of epithelial apical junctional proteins by a clathrin-mediated pathway into a unique storage compartment. Molecular biology of the cell 15(1): 176-188.

15. Singh AB, Sharma, Dhawan P (2010) Claudin family of proteins and cancer: an overview. Journal of oncology 541957-541957.

16. Guarino M, Rubino, Ballabio (2007) The role of epithelial Mesenchymal transition in cancer pathology. Pathology 39(3): 305-318.

17. Rendon Huerta E, Chavarria Velazquez C, Montaño L, Claudins (2013) Inflammation and Epithelial-Mesenchymal Transition in Gastric Tissue. Journal of Gastrointestinal \& Digestive System.

18. Bhat AA, Washington $M K$, Beauchamp RD, Singh AB, Dhawan $P$, et al. (2015) Claudin 7 expression induces mesenchymal to epithelial transformation (MET) to inhibit colon tumorigenesis. Oncogene 34(35): 4570-4580.

19. Luissint AC, Parkos, Nusrat A (2016) Inflammation and the Intestinal Barrier: Leukocyte-Epithelial Cell Interactions, Cell Junction Remodeling, and Mucosal Repair. Gastroenterology 151(4): 616-632.

20. Anderson SM (2015) Chapter 46 - Lactation and its Hormonal Control, in Knobil and Neill's Physiology of Reproduction. ( $4^{\text {th }}$ Edn).

21. Pope JL, Washington MK, Beauchamp RD, Singh AB, Dhawan P, et al. (2014) Claudin-1 regulates intestinal epithelial homeostasis through the modulation of Notch-signalling. Gut 63(4): 622-634.

22. Dhawan P, Schmidt C, Neff J, Washington MK, Beauchamp RD, et al. (2005) Claudin-1 regulates cellular transformation and metastatic behavior in colon cancer. The Journal of clinical investigation 115(7): $1765-1776$

23. Zeissig S, Kroesen, Zeitz M, Fromm M, Schulzkeet, et al. (2007) Changes in expression and distribution of claudin 2, 5 and 8 lead to discontinuous tight junctions and barrier dysfunction in active Crohn's disease. Gut 56(1): 61-72.

24. Ahmad R, Mary K, Washington, Luc Van Kaer, Punita Dhawan, et al. (2014) Targeted colonic claudin-2 expression renders resistance to epithelial injury, induces immune suppression, and protects from colitis. Mucosal Immunology 7: 1340.

25. Ding L, Lu Q Renegar R, Cao J, Chen YH, et al. (2012) Inflammation and disruption of the mucosal architecture in claudin-7-deficient mice. Gastroenterology 142(2): 305-315.

26. Tanaka H, Kiyonari H, Shioi G, Tamura A, Tsukita S, et al. (2015) Intestinal deletion of em Claudin-7enhances paracellular organic solute flux and initiates colonic inflammation in mice. Gut 64(10): 1529. 
27. Tamura A, Wada M, Noda T, Watanabe M, Suzuki Y, et al. (2011) Loss of Claudin-15, but Not Claudin-2, Causes Na+ Deficiency and Glucose Malabsorption in Mouse Small Intestine. Gastroenterology 140(3): 913 923.

28. Tamura A (2008) Megaintestine in Claudin-15 Deficient Mice. Gastroenterology 134(2): 523-534.

29. Fujita K, Katahira J, Horiguchi Y, Sonoda N, Furuse M, et al. (2000) Clostridium perfringens enterotoxin binds to the second extracellular loop of claudin-3, a tight junction integral membrane protein. FEBS Letters 476(3): 258-261.

30. Fernández Miyakawa ME, Pistone Creydt, Uzal Mc Clane, Ibarra (2005) Clostridium perfringens enterotoxin damages the human intestine in vitro. Infection and immunity 73(12): 8407-8410.

31. Veshnyakova A (2010) On the interaction of Clostridium perfringens enterotoxin with claudins. Toxins 2(6): 1336-1356.

32. Saitoh Y (2015) Structural insight into tight junction disassembly by \&lt; em\&gt; Clostridium perfringens\&lt;/em\&gt; enterotoxin. Science 347(6223): 775 .

33. Evans MJ, Wölk B, Hatziioannou T, Mc Keating JA, Bieniasz PD, et al. (2007) Claudin-1 is a hepatitis $C$ virus co-receptor required for a late step in entry. Nature 446(7137): 801-805.

ISSN: 2574-1241

DOI: 10.26717/BJSTR.2019.21.003637

Sabah Nisar, Sheema Hashem. Biomed J Sci \& Tech Res

(C) This work is licensed under Creative Commons Attribution 4.0 License

Submission Link: https://biomedres.us/submit-manuscript.php
34. Fedwick JP, Hatziioannou T, Mc Keating JA, Bieniasz PD, Rice CM (2005) Helicobacter pylori activates myosin light-chain kinase to disrupt claudin- 4 and claudin-5 and increase epithelial permeability. Infection and immunity 73(12): 7844-7852.

35. Tanaka M, Kamata R, Sakai R (2005) Phosphorylation of ephrin-B1 via the interaction with claudin following cell-cell contact formation. The EMBO journal 24(21): 3700-3711.

36. Vancamelbeke M, Vermeire S (2017) The intestinal barrier: a fundamental role in health and disease. Expert review of gastroenterology \& hepatology 11(9): 821-834.

37. Grootjans J, Geertje Thuijls, Froukje Verdam, Joep PM Derikx, Kaatje Lenaerts, et al. (2010) Non-invasive assessment of barrier integrity and function of the human gut. World journal of gastrointestinal surgery 2(3): 61-69.

38. Hollandsworth HM (2019) Anti-Claudin-1 Conjugated to a Near-Infrared Fluorophore Targets Colon Cancer in PDOX Mouse Models. Journal of Surgical Research 242: 145-150.

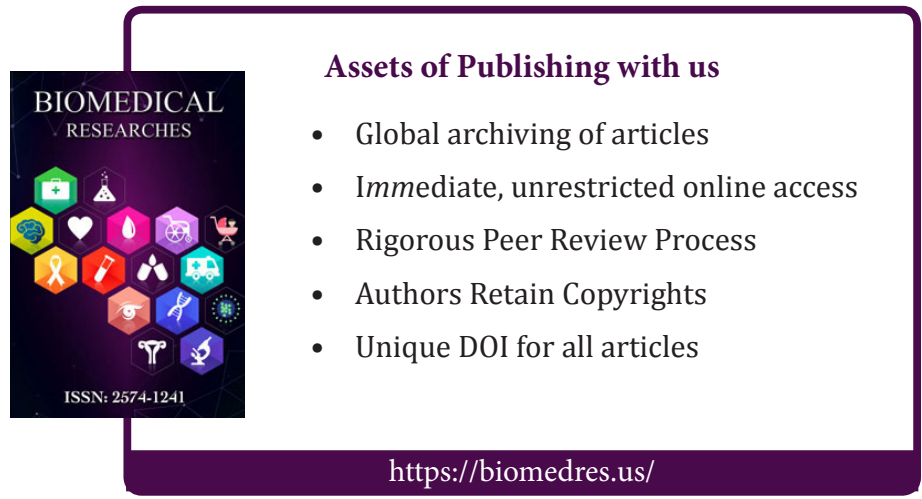

